\title{
Inhibition of Multidrug Transporter in Tumor Endothelial Cells Enhances Antiangiogenic Effects of Low-Dose Metronomic Paclitaxel
}

Kosuke Akiyama, ${ }^{* \dagger}$ Nako Maishi, ${ }^{* \dagger}$ Noritaka Ohga, ${ }^{\dagger}$ Yasuhiro Hida, ${ }^{\ddagger}$ Yusuke Ohba, ${ }^{\S}$ Mohammad Towfik Alam, ${ }^{\dagger \uparrow}$ Taisuke Kawamoto, ${ }^{\dagger}$ Hitomi Ohmura, ${ }^{\dagger}$ Kenji Yamada, ${ }^{* \dagger}$ Chisaho Torii, ${ }^{* \dagger}$ Masanobu Shindoh, ${ }^{\top}$ and Kyoko Hida*†

From the Division of Vascular Biology, ${ }^{*}$ Institute for Genetic Medicine, the Departments of Vascular Biology ${ }^{\dagger}$ and Oral Pathology and Biology, ${ }^{\Uparrow}$ Graduate School of Dental Medicine, and the Departments of Cardiovascular and Thoracic Surgery ${ }^{\ddagger}$ and Cell Physiology, ${ }^{\S}$ Graduate School of Medicine, Hokkaido University, Sapporo, Japan

Accepted for publication

October 9, 2014.

Address correspondence to Kyoko Hida, D.D.S., Ph.D., Division of Vascular Biology, Institute for Genetic Medicine, Hokkaido University, N15 W7, Kita-ku, Sapporo 060-0815, Japan.

E-mail: khida@igm.hokudai. ac.jp.

\begin{abstract}
Tumor angiogenesis plays an important role in tumor progression and metastasis. Tumor endothelial cells (TECS) are a therapeutic target of antiangiogenic chemotherapy that was recently developed and is currently being investigated in the clinic with promising results. Low-dose chemotherapy, which is the long-term administration of relatively low doses of chemotherapeutic agents, has been proposed for targeting tumor angiogenesis in various types of cancers. Although the efficacy of low-dose chemotherapy has been confirmed in several clinical models, some studies show insufficient therapeutic effect for malignant cancers. As a possible mechanism of the treatment failure, it has been considered that tumor cells may acquire resistance to this therapy. However, drug resistance by TECs may also be due to another mechanism for resistance of tumor cells to low-dose chemotherapy. We reported elsewhere that TECs were resistant to the anticancer drug paclitaxel, which is a mitotic inhibitor, concomitant with P-glycoprotein up-regulation. Verapamil, a P-glycoprotein inhibitor, abrogated TEC resistance in vitro. Herein, we demonstrated that verapamil coadministration enhanced the effects of low-dose paclitaxel concomitant with inhibiting tumor angiogenesis in a preclinical in vivo mouse melanoma xenograft model. Furthermore, verapamil coadministration reduced lung metastasis. These results suggest that inhibiting P-glycoprotein in TECs may be a novel strategy for low-dose chemotherapy targeting TECs. (Am J Pathol 2015, 185: 572-580; http://dx.doi.org/10.1016/j.ajpath.2014.10.017)
\end{abstract}

After the role of angiogenesis in tumor progression was first recognized, antiangiogenic chemotherapy that targeted tumor blood vessels was developed. ${ }^{1}$ Current antiangiogenic drugs, such as bevacizumab, that target vascular endothelial growth factor (VEGF) have shown a good therapeutic effect. However, these drugs produce some positive effects in patients with specific cancer types when bevacizumab is combined with conventional chemotherapy. In addition, it has been reported that drug resistance to antiangiogenic chemotherapy emerged because tumor cells can induce angiogenesis with compensatory secretion of other angiogenic growth factors, such as fibroblast growth factor-2 and angiopoietin, when VEGF is inhibited. ${ }^{2}$

Anticancer drugs target highly proliferative cells, such as tumor cells. Anticancer drugs have generally been administered in a short cycle with prolonged drug-free breaks at the maximum tolerated dose. Unfortunately, the high-dose anticancer drugs frequently cause substantial toxicity, resulting in adverse effects that might limit the treatment.

It was recently suggested that cytotoxic anticancer agents could target tumor vasculature because tumor endothelial cells (TECs) are more proliferative compared with normal endothelial cells (NECs). ${ }^{3}$ Low-dose chemotherapy, which is called metronomic chemotherapy, is one of the antiangiogenic therapies. This therapy targets proliferating TECs with long-term administration of chemotherapeutic agents, such as the mitotic inhibitor paclitaxel and the DNA synthesis inhibitor 5-fluorouracil (5-FU), which show cytotoxic action on proliferating TECs at relatively low, minimally toxic doses to reduce

Supported by Grants-in-Aid for Scientific Research from the Ministry of Education, Science, and Culture of Japan (grant 23112501 to K.H.) and The Naito Foundation (K.H.).

Disclosures: None declared. 
adverse effects. ${ }^{4-6}$ Several benefits of low-dose chemotherapy have been reported compared with anti-VEGF therapy and maximum tolerated dose treatment. Low-dose chemotherapy can i) reduce undesirable adverse effects caused by myelosuppression because the dose of anticancer drugs is low or by damage to NECs because it acts specifically on TECs; ii) inhibit tumor regrowth caused by the resuming of tumor angiogenesis because it does not need prolonged drug-free breaks, unlike conventional chemotherapy, such as treatment at the maximum tolerated dose; and iii) inhibit tumor angiogenesis in several aspects, such as inhibition of proliferating TECs, inducing up-regulation of the endogeneous angiogenesis inhibitor thrombospondin- $1,{ }^{7}$ and inhibition of endothelial progenitor cell mobilization from the bone marrow.

Clinical trials with metronomic chemotherapy were undertaken for several types of solid tumors, including advanced breast cancer $^{8-11}$ and glioblastoma. ${ }^{12-14}$ Promising results were obtained for malignancies that were poorly responsive to conventional chemotherapies.

However, low-dose chemotherapy did not improve patient survival in some cases. It is believed that tumor cells acquire drug resistance as the mechanism for the treatment failure. However, the detailed mechanism still remains unknown.

Recent studies have revealed that TECs were drug resistant compared with NECs, contrary to the traditional assumption that TECs are genetically stable and do not acquire drug resistance. ${ }^{15}$ We also reported that TECs had cytogenetic abnormalities, such as aneuploidy in mouse tumors ${ }^{16}$ and human renal carcinomas. ${ }^{17}$ In addition, TECs are resistant to certain anticancer drugs, such as paclitaxel. ${ }^{18,19}$ Recently, we found that TECs were resistant to paclitaxel concomitant with up-regulation of MDRI (multidrug-resistance gene)/P-glycoprotein (P-gp) in the tumor microenvironment. ${ }^{18}$ Furthermore, the resistance to paclitaxel by cultured TECs in vitro was abrogated by a P-gp inhibitor, verapamil.

P-gp, a member of the $\mathrm{ABC}$ transporter family, is a transmembrane glycoprotein and a multidrug transporter. A variety of studies have reported that $M D R 1 / \mathrm{P}$-gp played a major role in drug resistance to several anticancer drugs. ${ }^{20-22}$ The results of a previous study suggested that even TECs could acquire resistance to antiangiogenic chemotherapy concomitant with their up-regulation of P-gp. ${ }^{18}$ Thus, we hypothesized that inhibiting P-gp in TECs may improve the response to antiangiogenic chemotherapy.

Herein, we investigated the antiangiogenic efficacy of coadministering the P-gp inhibitor verapamil along with low-dose metronomic paclitaxel using an in vivo human melanoma model in mice.

\section{Materials and Methods}

\section{Cell Line and Culture Conditions}

A375SM cells (human highly metastatic melanoma cells) were a gift from Dr. Isaiah J. Fidler (MD Anderson Cancer Center, Houston, TX) ${ }^{23}$ in 2007 and were authenticated in
January 2014 by JCRB Cell Bank (Osaka, Japan) by short tandem repeat analysis. These cells were cultured in minimal essential medium (Gibco, Grand Island, NY) supplemented with $10 \%$ fetal bovine serum in a humidified atmosphere with $5 \% \mathrm{CO}_{2}$ and $95 \%$ air at $37^{\circ} \mathrm{C}$.

\section{Antibodies}

The following antibodies were used: Alexa Fluor 647 rat antimouse CD31 (BioLegend, San Diego, CA), rabbit antimouse/human MDRI/P-gp (LifeSpan Biosciences Inc., Seattle, WA), rabbit anti-mouse/human cleaved caspase3 (Cell Signaling Technology Inc., Beverly, MA), Alexa Fluor 594 goat anti-rabbit IgG (Life Technologies Inc., Gaithersburg, MD), and monoclonal anti- $\beta$-actin (AC-15; SigmaAldrich, St. Louis, MO).

\section{Isolation of TECs and NECS}

TECs were isolated from melanoma xenografts in nude mice, and NECs were isolated from mouse dermis as described elsewhere. ${ }^{16,24}$ All the animal experimental procedures were approved by the local animal research authorities, and animal care was in accordance with institutional guidelines. In brief, excised tissues were minced and digested with collagenase II. After blood cells were removed by a single sucrose step-gradient centrifugation using Histopaque 1077, cell suspensions were filtered, and endothelial cells (ECs) were isolated using a magnetic cell sorting system (Miltenyi Biotec, Tokyo, Japan) with anti-mouse CD31 antibodies. CD31positive cells were sorted and plated on fibronectincoated culture plates and were grown in EGM-2MV medium (Lonza, Basel, Switzerland) and $15 \%$ fetal bovine serum. Diphtheria toxin $(500 \mathrm{ng} / \mathrm{mL}$; Calbiochem, San Diego, CA) was added to TEC subcultures to eliminate human tumor cells and to NEC subcultures for technical consistency. After subculture for approximately 2 weeks, isolated ECs were further purified using FITCBS1-B4-lectin. ${ }^{25}$ All the purified ECs were cultured in EGM-2MV and were used at passages 15 to 25 .

\section{Immunostaining}

Tumor tissues were dissected out from humanely sacrificed mice. Frozen sections of excised tissues were prepared as described elsewhere. ${ }^{26}$ To assess P-gp co-localization in tumor blood vessels, frozen sections were double stained with rat anti-mouse CD31-Alexa Fluor 647 and rabbit anti$M D R 1 / \mathrm{P}-\mathrm{gp}$ antibodies, followed by counterstaining with DAPI to stain nuclei. To detect apoptotic ECs, frozen sections were double stained with rat anti-mouse CD31-Alexa Fluor 647 and anti-cleaved caspase 3 antibodies. Stained samples were observed using a FluoView FV10i confocal microscope (Olympus America Inc., Center Valley, PA). Cleaved caspase 3 -positive proportions in blood vessels were quantified 
using ImageJ software version $1.43 \mathrm{u}$ (NIH, Bethesda, MD). Quantitative analysis was performed using five separate images in each tumor section. Three to four mice per group were used for the in vivo experiments.

\section{Microvascular Density Analysis}

Mice were anesthetized and tumors were resected on day 52 . Frozen sections were prepared, and the microvascular density of each CD31-stained tumor was determined in five randomly selected fields ( $n=3$ to 4 mice per group). The percentage of CD31-stained area was analyzed in five randomly selected fields using ImageJ software.

\section{Cell Survival Assay}

TECs and NECs were seeded in triplicate at a density of $5 \times 10^{3}$ cells per well in a 96-well plate containing EGM-2MV and were washed after 6 hours. These cells were treated with paclitaxel (Taxol; Biomol, Plymouth Meeting, PA), verapamil (Taiyo, Osaka, Japan), diltiazem (Sigma-Aldrich), or amlodipine (Sigma-Aldrich) at the indicated concentrations in EGM$2 \mathrm{MV}$ for 72 hours. Cell viability was assessed using a 3-(4,5-dimethylthiazol-2-yl)-5-(3-carboxymethoxyphenyl)2-(4-sulfophenyl)-2H-tetrazolium inner salt assay (Promega Corp, Tokyo, Japan).

\section{Quantitative Real-Time RT-PCR}

Total RNA was extracted using an RNeasy micro kit (Qiagen Inc., Valencia, CA). cDNA was synthesized using ReverTraPlus (Toyobo Co., Osaka, Japan), as described elsewhere. ${ }^{26}$ cDNA was amplified by PCR. The PCR products were visualized by ethidium bromide staining and UV transillumination. Real-time RT-PCR was performed using SsoFast EvaGreen supermix (Bio-Rad Laboratories, Hercules, CA). Cycling conditions were according to the manufacturer's instructions based on the use of CFX Manager software version 3.0 (BioRad Laboratories). Relative expression levels were determined after normalization to glyceraldehyde-3-phosphate dehydrogenase mRNA expression. The following primers were used: mouse glyceraldehyde-3-phosphate dehydrogenase, $5^{\prime}$-TCTGACGTGCCGCCTGGAG-3' (forward) and 5'-TCGCAGGAGACAACCTGGTC- $3^{\prime}$ (reverse) and mouse multidrug resistance 1 (Mdr1), 5'-ATCCGGGAGCAGAAGTTTGA-3' (forward) and $5^{\prime}$-GCACCAAAGACAACAGCAGA-3' (reverse).

\section{Western Blot Analysis}

Cells were lyzed as previously described. ${ }^{26}$ Total protein concentration was determined using a BCA protein assay kit (Pierce Biotechnology, Rockford, IL). Western blot analysis was performed using antibodies specific for P-gp and $\beta$-actin and horseradish peroxidase-conjugated secondary antibodies, as described elsewhere. ${ }^{27} \mathrm{P}$-gp levels were normalized to $\beta$-actin levels by scanning densitometry using ImageJ software.

\section{Plasmids and Transfection}

cDNA for Rluc (a gift from Dr. Yoshihiro Ohmiya, National Institute of Advanced Industrial Science and Technology, Ikeda, Japan) was amplified by PCR and was cloned into pCR-Blunt II-TOPO (Invitrogen, Carlsbad, CA). The coding sequence for Rluc was subcloned into the XhoI and NotI sites of pCAGGS-Venus, ${ }^{28}$ and the DNA fragment encoding for Venus and Rluc was then subcloned into the EcoRI and NotI sites of pCSII-CMVMCS (a gift from Dr. Hiromi Miyoshi, RIKEN, Wako, Japan). This self-inactivating lentivirus vector together with the packaging vector pCAG-HIVgp and the VSV-Gand REV-expressing construct pCMV-VSV-G-RSV-REV (a gift from Dr. Hiromi Miyoshi) were introduced into 293T cells using FuGENE HD transfection reagent (Roche Diagnostics GmbH, Mannheim, Germany), according to the manufacturer's recommendations. Lentivirus-mediated gene transfer was performed as described elsewhere. ${ }^{29}$

\section{Mouse Tumor Xenograft Model and Treatment Regimens}

Six-week-old female nude mice (BALB/c Slc-nu/nu) were acquired from Sankyo Labo Service Corp. (Tokyo, Japan) and were housed under specific pathogen-free conditions. All the procedures for animal care and experimentation adhered to institutional guidelines and were approved by the local animal research authorities. Rluc-transfected A375SM cells $\left(1 \times 10^{6}\right)$ were subcutaneously implanted in the right flanks of nude mice. When tumors reached an average size of $200 \mathrm{~mm}^{3}$, the following treatments were initiated: sterile Hanks' balanced salt solution (HBSS) (i.p. twice weekly, control), verapamil $(20 \mathrm{mg} / \mathrm{kg}$ i.p. twice weekly), low-dose metronomic paclitaxel (7.5 mg/kg i.p. twice weekly), low-dose metronomic paclitaxel and verapamil (paclitaxel: $7.5 \mathrm{mg} / \mathrm{kg}$ and verapamil: 20 $\mathrm{mg} / \mathrm{kg}$ i.p. twice weekly), low-dose metronomic 5-FU (10 mg/ $\mathrm{kg}$ i.p. twice weekly), and low-dose metronomic 5FU + verapamil (5-FU: $10 \mathrm{mg} / \mathrm{kg}$ i.p. and verapamil: 20 $\mathrm{mg} / \mathrm{kg}$ i.p. twice weekly). For these experiments, three to four mice per group were used. Mice were monitored regularly. Tumor volume was measured using the following standard formula: (shortest diameter $)^{2} \times($ longest diameter $) \times 0.5$.

No differences were observed in body weight among these groups throughout the experimental period. After 37 days of treatment, tumors were excised. After the mice were sacrificed, their lungs were resected and subjected to ex vivo bioluminescence imaging using the IVIS Spectrum system (Caliper Life Science, Hopkinton, MA). Bioluminescent signals were quantified using Living Image software version 3.0 (Caliper Life Science). The number of lungs with bioluminescent signals was counted and is indicated by a percentage in each group. 


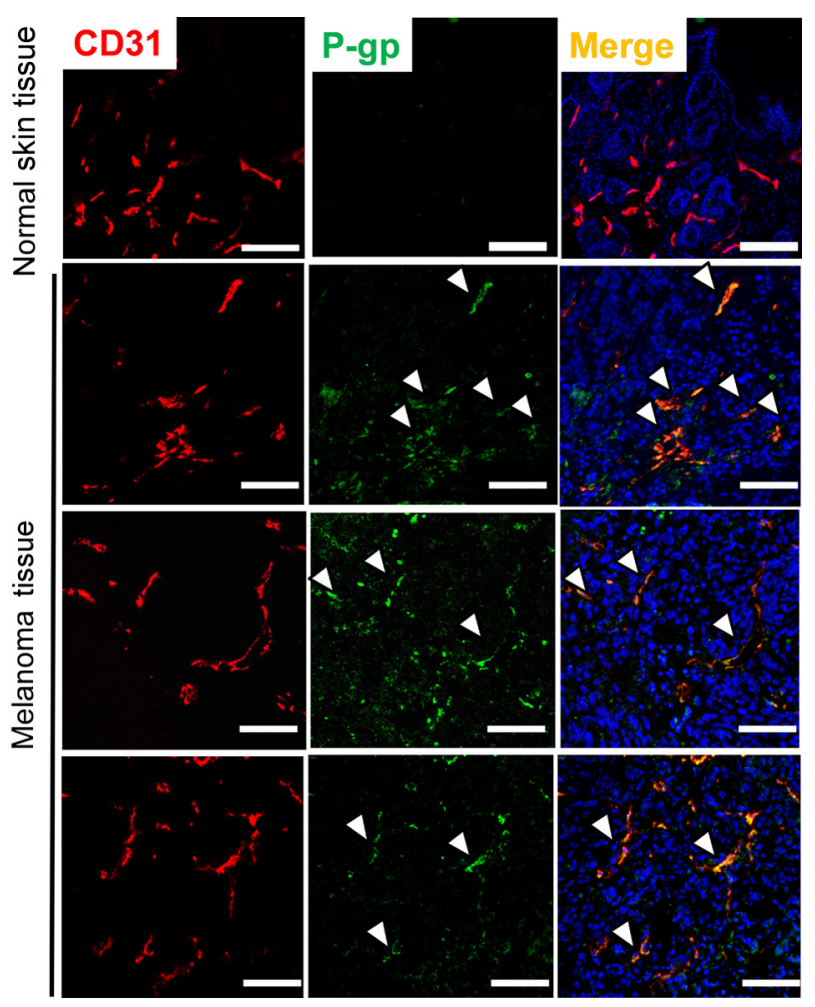

Figure 1 P-glycoprotein (P-gp) is up-regulated in tumor endothelium in vivo. Immunostaining for the endothelial markers CD31 and P-gp in tumors (A375SM) and in mouse normal tissues (dermal tissue). Merged staining (white arrowheads) shows co-localization of P-gp (green) and CD31 (red). Nuclei were stained blue (DAPI). Scale bars: $100 \mu \mathrm{m}$.

\section{Statistical Analysis}

Results are given as means $\pm \mathrm{SD}$. Group comparisons were made by Mann-Whitney $U$-test for continuous variables. Comparisons within groups at each time point were made by paired Student's $t$-tests. $P<0.05$ and $P<0.01$ were considered statistically significant.

\section{Results}

\section{P-gp Is Up-regulated in Tumor Endothelium in Vivo}

To assess P-gp expression in the tumor blood vessels of A375SM tumor xenografts in mice, fluorescent double immunostaining was performed using anti-P-gp and antiCD31 antibodies. P-gp was mainly expressed in tumor blood vessels, whereas it was barely expressed in normal blood vessels in the dermis of control mice (Figure 1). P-gp expression in the blood vessels of A375 xenografted tumors (lower-grade malignancy than A375SM) was analyzed to determine whether P-gp expression in tumor blood vessels differs depending on tumor type or malignancy. P-gppositive TECs were present in tumor blood vessels in A375 tumors, but the number of P-gp-expressing tumor blood vessels was lower in A375 tumors than in A375SM tumors (Supplemental Figure S1A). Moreover, significantly more
P-gp-positive areas were present in tumor blood vessels of A375SM tumors than in blood vessels of A375 tumors $(P<0.01)$ (Supplemental Figure S1B). These results suggest that P-gp is expressed in TECs of several tumors but that its expression level may differ among tumors. P-gp was minimally expressed in A375SM cells versus tumor blood vessels. This finding suggested that TECs may be resistant to anticancer drugs that are P-gp substrates, including paclitaxel, a mitotic inhibitor that prevents cell division.

\section{The P-gp Inhibitor Verapamil Abrogates TEC Drug Resistance to Paclitaxel in Vitro}

As reported elsewhere, ${ }^{18}$ TECs isolated from A375SM melanomas were resistant to paclitaxel concomitant with up-regulated $M D R 1 / \mathrm{P}$-gp expression. Consistent with the previous report, freshly isolated TECs exhibited resistance to paclitaxel compared with NECs as determined by the 3-(4, 5-dimethylthiazol-2-yl)-5-(3-carboxymethoxyphenyl)-2(4-sulfophenyl)-2H-tetrazolium inner salt assay $(P<0.01)$ (Figure 2A). Mdr1 mRNA expression levels were approximately threefold higher in TECs than in NECs $(P<0.01)$ (Figure 2B). Resistance to paclitaxel and Mdr1 mRNA expression were analyzed between TECs isolated from A375 melanomas and TECs from A375SM melanomas to confirm whether the drug resistance phenotype is different between ECs. A375SM TECs were more resistant to paclitaxel than were other ECs (Supplemental Figure S2A). Mdr1 expression levels in

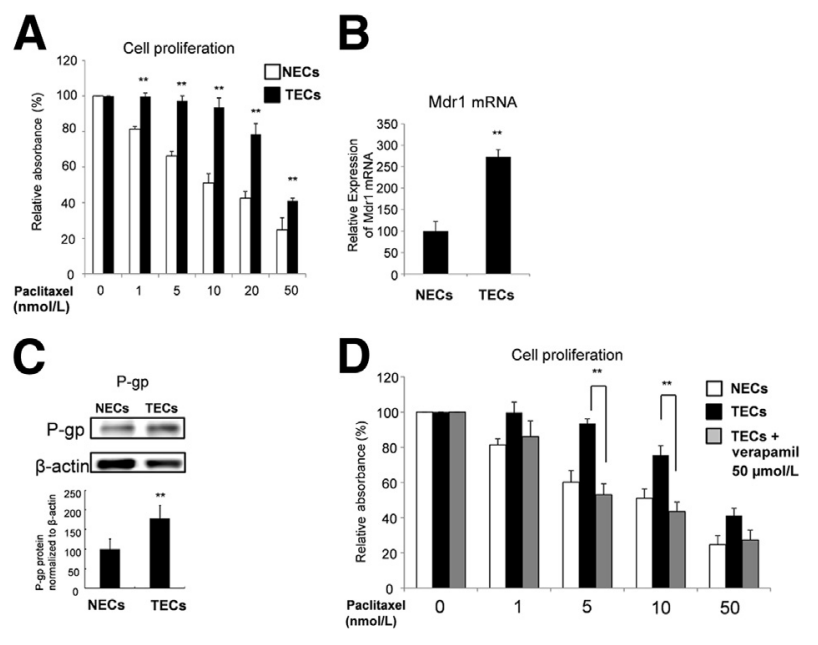

Figure 2 The P-glycoprotein (P-gp) inhibitor verapamil abrogates drug resistance by tumor endothelial cells (TECs) in vitro. A: Sensitivity to paclitaxel was assessed by the 3-(4,5-dimethylthiazol-2-yl)-5-(3-carboxymethoxyphenyl)2-(4-sulfophenyl)-2H-tetrazolium inner salt (MTS) assay after exposing cells to this drug for 72 hours. Melanoma endothelial cells (ECs) were more resistant to paclitaxel than were normal ECs (NECS). B: Mouse multidrug resistance 1 (Mdr1) mRNA expression was determined by real-time PCR. Mdr1 mRNA expression in melanoma ECs was significantly higher than that in NECs. C: P-gp protein expression was determined using Western blot analysis. P-gp protein expression in melanoma ECs was significantly higher than that in NECs. D: Sensitivity to paclitaxel in conjunction with verapamil treatment was determined by the MTS assay after exposure for 72 hours. Melanoma ECs were resensitized to paclitaxel by verapamil. Data are given as means \pm SD. ${ }^{* *} P<0.01$. 


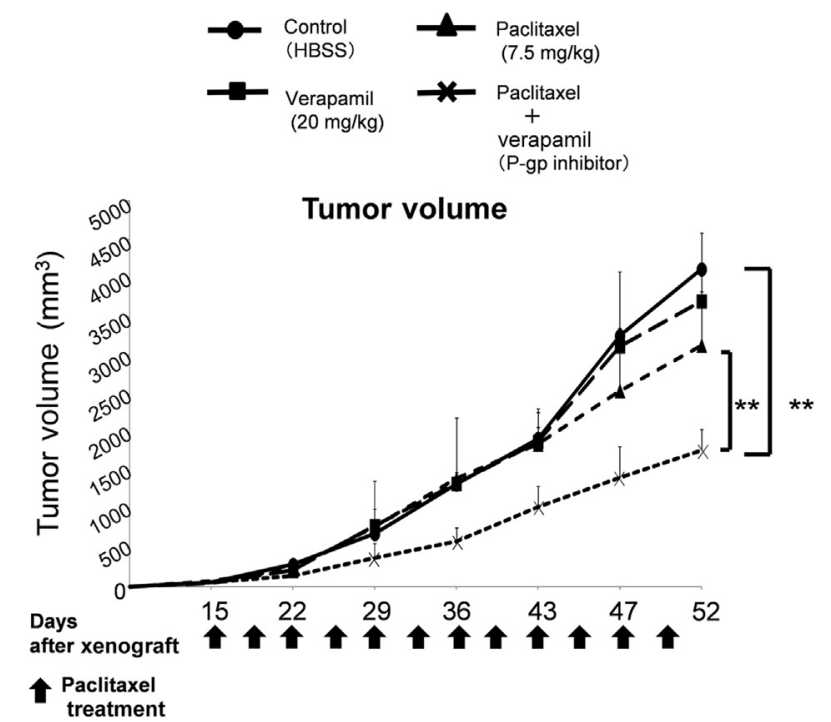

Figure 3 Verapamil enhances metronomic chemotherapy effects in vivo. After tumor establishment, mice were treated according to the following low-dose metronomic schedule for 52 days: sterile Hanks' balanced salt solution (HBSS) (i.p. twice weekly, control), verapamil $(20 \mathrm{mg} / \mathrm{kg}$ i.p. twice weekly), low-dose metronomic paclitaxel $(7.5 \mathrm{mg} /$ kg i.p. twice weekly), or low-dose metronomic paclitaxel + verapamil (paclitaxel: $7.5 \mathrm{mg} / \mathrm{kg}$ and verapamil: $20 \mathrm{mg} / \mathrm{kg}$ i.p. twice weekly). Tumor volumes were measured periodically. Verapamil enhanced the antitumor effects of paclitaxel. Data are given as means \pm SD. $* * P<0.01$.

A375SM TECs were higher than those in other ECs (Supplemental Figure S2B). These results suggest that drug resistance in TECs may depend on differences in the tumor microenvironment. P-gp protein expression levels in TECs were also higher than those in NECs $(P<0.01)$ (Figure 2C). The P-gp inhibitor verapamil, which is an L-type calcium channel blocker, restored the sensitivity of TECs to paclitaxel (Figure 2D).

To demonstrate that these inhibitory effects were caused by inhibiting P-gp and were not effects of an L-type calcium channel blocker on TECs, we assessed the drug sensitivity to paclitaxel in TECs along with two other drugs: a P-gp-inhibiting calcium channel blocker, diltiazem, and a non-P-gp-inhibiting calcium channel blocker, amlodipine. Diltiazem restored the sensitivity of TECs to paclitaxel, as did verapamil, whereas amlodipine did not (Supplemental Figure S3). These results suggested that coadministering a P-gp inhibitor with paclitaxel may enhance the antitumor effects of low-dose metronomic paclitaxel in vivo.

\section{Verapamil Enhances Metronomic Chemotherapy Effects}

To assess the efficacy of verapamil on low-dose metronomic paclitaxel treatment, we used verapamil in in vivo tumor experiments. After tumor establishment, mice were treated according to a low-dose metronomic schedule for 52 days. Tumor volumes were measured periodically.
Compared with control (HBSS) or verapamil treatment alone, administering paclitaxel alone inhibited tumor growth only slightly. There was no significant difference in tumor volumes among control (HBSS), verapamil alone, and paclitaxel alone (Figure 3). However, paclitaxel + verapamil significantly inhibited tumor growth compared with control (HBSS) or paclitaxel alone $(P<0.01)$ (Figure 3). Verapamil alone had no effect on tumor volume in this model.

To confirm whether the effects of verapamil were specific for P-gp, mice were treated with 5-FU, which is not a P-gp substrate and is not exported to the extracellular space by P-gp, using a low-dose metronomic schedule for the same amount of time. Coadministering verapamil had no additional antitumor effects when using 5-FU (Supplemental Figure S4). These results suggested that P-gp inhibition in TECs enhanced the cytotoxic activity of anticancer drugs that are P-gp substrates, such as paclitaxel.
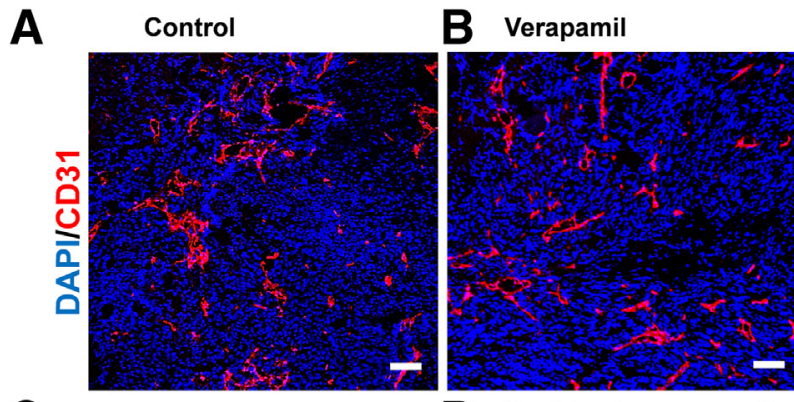

C
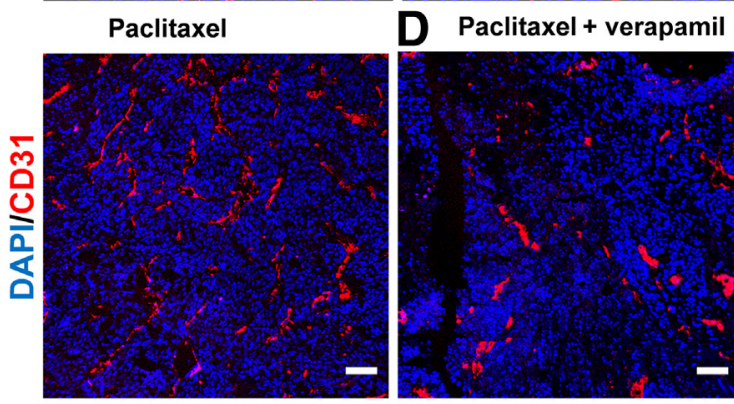

E

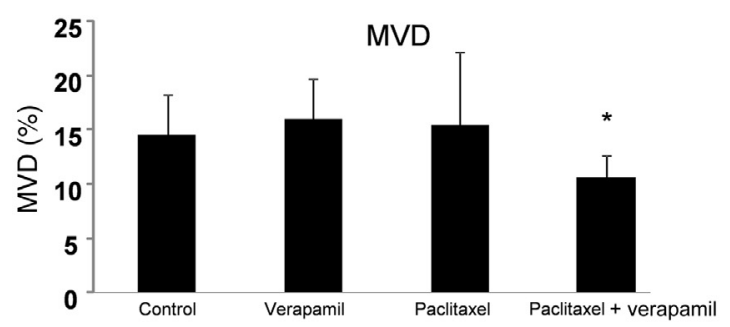

Figure 4 Verapamil enhances the antiangiogenic activity of low-dose metronomic paclitaxel in vivo. A-D: Immunohistochemical analysis for CD31 (red) was used for A375SM tumor sections from each group of mice after low-dose metronomic treatment. Nuclei were stained blue (DAPI). E: For microvascular density (MVD) analysis, the areas of vessels in CD31stained sections were determined. The percentage of CD31-stained area was analyzed in five randomly selected fields by using ImageJ software version $1.43 \mathrm{u}$ ( $\mathrm{NIH}$, Bethesda, MD). Data are given as means $\pm \mathrm{SD}$. ${ }^{*} P<0.05$. Scale bars: $100 \mu \mathrm{m}$. 


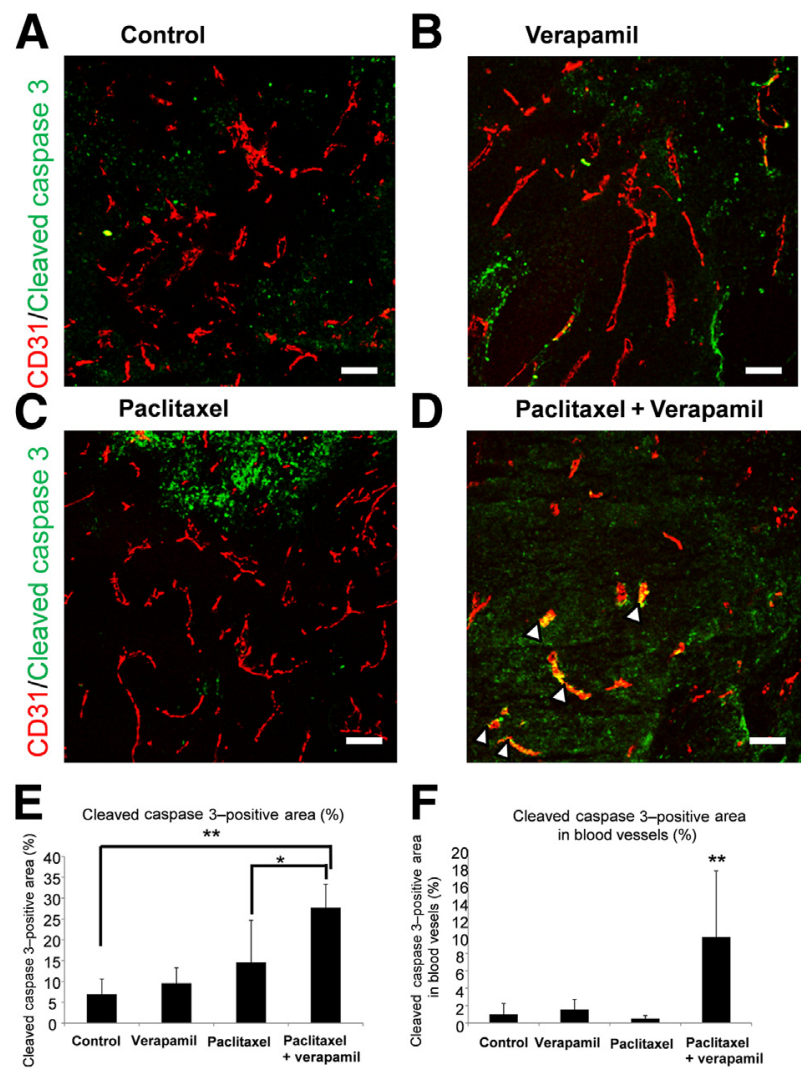

Figure 5 Verapamil coadministration induces tumor endothelial cell (TEC) apoptosis in vivo. A-D: Immunohistochemical analysis for CD31 (red) and cleaved caspase 3 (green) was used for A375SM tumor sections from each group of mice after low-dose metronomic treatment. Nuclei were stained blue (DAPI). Merged staining (white arrowheads) shows co-localization of cleaved caspase 3 (green) and CD31 (red). E: Cleaved caspase 3-positive areas were quantified using MetaMorph imaging software version 7.7.10 (Molecular Devices Inc., Sunnyvale, CA). Cleaved caspase 3-positive cells in tumor tissues were significantly increased in the paclitaxel + verapamil group compared with the other groups. F: Cleaved caspase 3-positive areas in tumor blood vessels were quantified using MetaMorph imaging software.Cleaved caspase 3-positive areas in CD31-positive blood vessels were significantly increased in the paclitaxel + verapamil group. Data are given as means \pm SD. ${ }^{*} P<0.05,{ }^{*} P<0.01$. Scale bars: $100 \mu \mathrm{m}$.

\section{Verapamil Coadministration Enhances the}

Antiangiogenic Activity of Low-Dose Metronomic Paclitaxel Treatment in an in Vivo Tumor Model

Next, to evaluate the effects of coadministering paclitaxel and verapamil on TECs, we used immunohistochemical analysis for CD31 in tumor sections from each group of mice. Paclitaxel alone did not decrease the density of intratumoral blood vessels compared with that in the control (HBSS) or verapamil alone, whereas paclitaxel + verapamil decreased the density of intratumoral blood vessels (Figure 4, A-D). To quantify these antiangiogenic effects, we assessed microvascular density. Microvascular density analysis showed that tumor angiogenesis was significantly inhibited in the paclitaxel + verapamil group (means \pm SD: $10.55 \% \pm 2.08 \%$ ) compared with the other groups (control:
$14.54 \% \pm 3.72 \%$, verapamil: $15.96 \% \pm 3.76 \%$, and paclitaxel: $15.41 \% \pm 6.76 \%$; all $P<0.01$ ) (Figure 4E). These results suggested that P-gp inhibition in TECs enhanced the antiangiogenic effects of low-dose metronomic paclitaxel.

\section{Verapamil Coadministration Induces TEC Apoptosis}

It is known that metronomic chemotherapy targets proliferating TECs, which results in tumor regression. ${ }^{5}$ To evaluate apoptotic areas in tumor tissues after coadministering paclitaxel and verapamil, we used immunohistochemical analysis for cleaved caspase 3 and CD31 in tumor sections from each group of mice (Figure 5, A-D). Cleaved caspase 3 staining showed that apoptotic areas were slightly increased with paclitaxel alone compared with control or verapamil alone. Moreover, cleaved caspase 3-positive areas were significantly increased in the paclitaxel + verapamil group (Figure 5E), which suggested that verapamil had enhanced the antitumor effects of paclitaxel. We also evaluated TEC apoptosis, as shown in CD31 and cleaved caspase 3 double-positive areas, for each group.

The apoptotic areas in tumor blood vessels were significantly increased with paclitaxel + verapamil compared with the other groups (Figure 5, D and F), which suggested that verapamil coadministration had induced TEC apoptosis. These results were consistent with the microvascular density

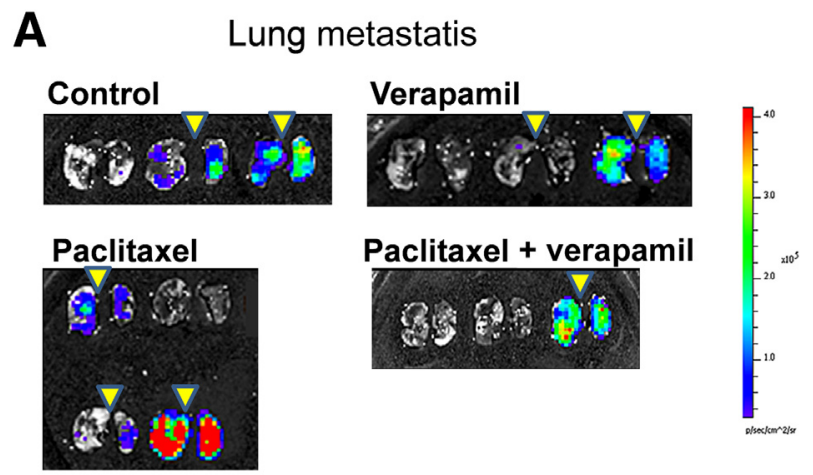

B

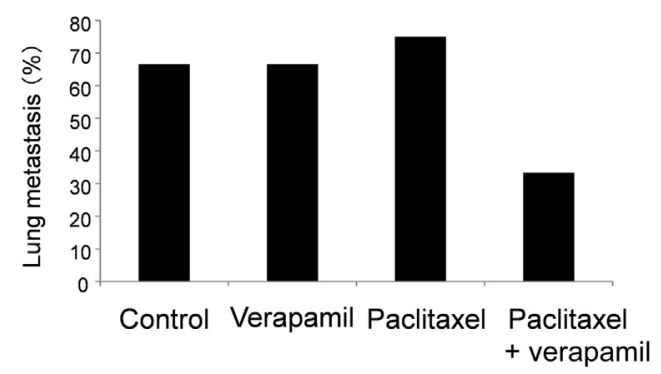

Figure 6 Verapamil reduces tumor metastasis to the lung. A: After treatment with a low-dose metronomic schedule for 52 days, the lungs were harvested from mice. The luminescence intensities of A375SM tumor cells in the lung (yellow arrowheads) were analyzed by the IVIS Spectrum imaging system (Caliper Life Science, Hopkinton, MA). B: The number of lungs with bioluminescent signals was counted in each group. The percentage on the $y$ axis represents luminescence-positive lungs in each group. 
results. Taken together, this suggested that P-gp inhibition in TECs by verapamil enhanced the antitumor effects of low-dose metronomic paclitaxel by inducing cellular apoptosis in tumor blood vessels.

\section{Verapamil Reduces Tumor Metastasis to the Lung}

It is known that tumor blood vessels are important for tumor metastasis. To assess the effects of verapamil coadministration on lung metastasis using the IVIS Spectrum imaging system, A375 tumor cells were transfected with lentiviral vectors that encoded for the luciferase-Venus gene before their inoculation into mice. After each treatment, lung tissues were resected from mice, and the luminescence intensities of A375 tumor cells in lung tissues were analyzed (Figure 6A). The number of lung metastases was decreased in the paclitaxel + verapamil group compared with the other groups, suggesting that verapamil coadministration reduced lung metastasis through its antiangiogenic effects (Figure 6B).

\section{Discussion}

In this study, we demonstrated for the first time that inhibiting P-gp in TECs with verapamil enhances the effects of lowdose chemotherapy (metronomic chemotherapy). Metronomic chemotherapy is one of the antiangiogenic therapies that involves long-term administration of chemotherapeutic agents at relatively low, minimally toxic doses without prolonged drug-free breaks. ${ }^{3}$ This type of chemotherapy targets proliferating TECs in addition to tumor cells, which results in inhibiting tumor angiogenesis.

To date, many clinical trials of metronomic chemotherapy have been conducted for several types of cancer, such as breast, ${ }^{10}$ ovarian, ${ }^{30}$ and prostate ${ }^{31}$ cancer. Although metronomic chemotherapy showed promising outcomes for several types of tumors, there were disappointing outcomes with metronomic paclitaxel for some tumors, such as metastatic melanoma. ${ }^{32}$ It has been reported that taxanes, including paclitaxel, have minimal efficacy for melanoma, and weekly paclitaxel treatment for previously treated patients resulted in no responses. ${ }^{33}$ However, no clear reasons for these negative results have been determined.

At present, antiangiogenic drugs, such as bevacizumab, sorafenib, and sunitinib, that target VEGF or VEGF receptors are used for cancer treatment. However, these therapeutic treatments occasionally lose their effectiveness over time for some tumors because tumor cells can acquire drug resistance with the compensatory secretion of growth factors. Similarly, the emergence of resistance to metronomic chemotherapy has been reported, possibly because of tumor malignancies that were poorly responsive to conventional chemotherapy. ${ }^{34,35}$ Vasculogenic mimicry is a tumor vascularization process associated with aggressive melanoma and other cancers characterized by tumor cells expressing EC markers. Furthermore, accepted antiangiogenic drugs did not work, partially because of their ineffectiveness against vasculogenic mimicry. ${ }^{36}$ However, vasculogenic mimicry was not considered a mechanism for resistance to antiangiogenic therapy in our A375SM case because no contamination of tumor cells occurred in the isolated TECs owing to the use of diphtheria toxin. ${ }^{16}$

It was recently reported that TECs were resistant to anticancer drugs compared with NECs ${ }^{15,37,38}$ and that TECs were cytogenetically unstable. ${ }^{16,17}$ Furthermore, we recently reported that TECs were resistant to paclitaxel concomitant with their enhanced expression of P-gp. ${ }^{18,19}$ Thus, we hypothesized that this drug resistance by TECs may also be another mechanism underlying the failure of metronomic chemotherapies.

$M D R 1 / \mathrm{P}-g p$ is a transmembrane glycoprotein that belongs to the ATP-binding cassette superfamily and functions as a multidrug transporter. It is well-known that P-gp overexpression in tumor cells causes their resistance to several anticancer drugs. ${ }^{20-22}$ The P-gp inhibitor verapamil is an L-type calcium channel blocker and is used for treating hypertension, angina pectoris, and cardiac arrhythmias. Verapamil has also been coadministrated with anticancer drugs to abolish the drug resistance by tumor cells. However, verapamil has never been used during metronomic chemotherapy.

We previously reported that TECs were resistant to the anticancer drug 5-FU, which is used in metronomic chemotherapy, and suggested that TECs could be resistant not only to paclitaxel but also to other anticancer drugs. ${ }^{19}$ However, verapamil did not exhibit any effects when it was coadministered with low-dose metronomic 5-FU. This suggested that the drug resistance by TECs was due not only to P-gp up-regulation. The results suggest that inhibiting P-gp in TECs by verapamil enhances the effects of anticancer drugs that are P-gp substrates.

In this study, coadministering paclitaxel and verapamil resulted in significant inhibition of tumor growth compared with paclitaxel administration alone, although it did not completely inhibit tumor growth. It is possible that the mechanism of TEC drug resistance is due not only to P-gp up-regulation because TECs are a heterogeneous population of cells. Indeed, A375SM TECs showed high MDRl expression and were remarkably resistant to paclitaxel compared with A375 TECs. This result suggests that these unique characteristics in each TEC may be caused by the tumor microenvironment. We previously reported that VEGF from tumor cells in the tumor microenvironment is a key factor for TEC drug resistance. ${ }^{18}$

Furthermore, as possible mechanisms for drug resistance by TECs, Akt activation ${ }^{37}$ and STAT3/MAPK activation ${ }^{15}$ in TECs have been reported. Additional studies are required to establish a more effective antiangiogenic strategy that targets drug-resistant TECs. 
In conclusion, to our knowledge, we demonstrated for the first time that the P-gp inhibitor verapamil enhances the antitumor effects of low-dose metronomic paclitaxel by abrogating drug resistance of tumor endothelium. A P-gp inhibitor may be useful for chemotherapy that targets TECs.

\section{Acknowledgments}

We thank Dr. Isaiah J. Fidler for providing the A375SM super-metastatic human malignant melanoma cell line and Dr. Aya Yanagawa, Tomomi Takahashi, and Yuko Suzuki for technical assistance.

\section{Supplemental Data}

Supplemental material for this article can be found at http://dx.doi.org/10.1016/j.ajpath.2014.10.017.

\section{References}

1. Folkman J: Tumor angiogenesis: therapeutic implications. N Engl J Med 1971, 285:1182-1186

2. Eikesdal HP, Kalluri R: Drug resistance associated with antiangiogenesis therapy. Semin Cancer Biol 2009, 19:310-317

3. Kerbel RS: Inhibition of tumor angiogenesis as a strategy to circumvent acquired resistance to anti-cancer therapeutic agents. Bioessays 1991, 13:31-36

4. Klement G, Baruchel S, Rak J, Man S, Clark K, Hicklin DJ, Bohlen P, Kerbel RS: Continuous low-dose therapy with vinblastine and VEGF receptor-2 antibody induces sustained tumor regression without overt toxicity. J Clin Invest 2000, 105:R15-R24

5. Browder T, Butterfield CE, Kraling BM, Shi B, Marshall B, O'Reilly MS, Folkman J: Antiangiogenic scheduling of chemotherapy improves efficacy against experimental drug-resistant cancer. Cancer Res 2000, 60:1878-1886

6. Hanahan D, Bergers G, Bergsland E: Less is more, regularly: metronomic dosing of cytotoxic drugs can target tumor angiogenesis in mice. J Clin Invest 2000, 105:1045-1047

7. Bocci G, Francia G, Man S, Lawler J, Kerbel RS: Thrombospondin 1, a mediator of the antiangiogenic effects of low-dose metronomic chemotherapy. Proc Natl Acad Sci U S A 2003, 100:12917-12922

8. Colleoni M, Rocca A, Sandri MT, Zorzino L, Masci G, Nole F, Peruzzotti G, Robertson C, Orlando L, Cinieri S, de BF, Viale G, Goldhirsch A: Low-dose oral methotrexate and cyclophosphamide in metastatic breast cancer: antitumor activity and correlation with vascular endothelial growth factor levels. Ann Oncol 2002, 13:73-80

9. Colleoni M, Orlando L, Sanna G, Rocca A, Maisonneuve P, Peruzzotti G, Ghisini R, Sandri MT, Zorzino L, Nole F, Viale G, Goldhirsch A: Metronomic low-dose oral cyclophosphamide and methotrexate plus or minus thalidomide in metastatic breast cancer: antitumor activity and biological effects. Ann Oncol 2006, 17: 232-238

10. Bottini A, Generali D, Brizzi MP, Fox SB, Bersiga A, Bonardi S, Allevi G, Aguggini S, Bodini G, Milani M, Dionisio R, Bernardi C, Montruccoli A, Bruzzi P, Harris AL, Dogliotti L, Berruti A: Randomized phase II trial of letrozole and letrozole plus low-dose metronomic oral cyclophosphamide as primary systemic treatment in elderly breast cancer patients. J Clin Oncol 2006, 24:3623-3628

11. Orlando L, Cardillo A, Ghisini R, Rocca A, Balduzzi A, Torrisi R, Peruzzotti G, Goldhirsch A, Pietri E, Colleoni M: Trastuzumab in combination with metronomic cyclophosphamide and methotrexate in patients with HER-2 positive metastatic breast cancer. BMC Cancer 2006, 6:225

12. Kong DS, Lee JI, Kim WS, Son MJ, Lim do H, Kim ST, Park K, Kim JH, Eoh W, Nam DH: A pilot study of metronomic temozolomide treatment in patients with recurrent temozolomide-refractory glioblastoma. Oncol Rep 2006, 16:1117-1121

13. Perry JR, Rizek P, Cashman R, Morrison M, Morrison T: Temozolomide rechallenge in recurrent malignant glioma by using a continuous temozolomide schedule: the "rescue" approach. Cancer 2008, 113:2152-2157

14. Reardon DA, Desjardins A, Vredenburgh JJ, Gururangan S, Sampson JH, Sathornsumetee S, McLendon RE, Herndon JE 2nd, Marcello JE, Norfleet J, Friedman AH, Bigner DD, Friedman HS: Metronomic chemotherapy with daily, oral etoposide plus bevacizumab for recurrent malignant glioma: a phase II study. Br J Cancer 2009, 101:1986-1994

15. Xiong YQ, Sun HC, Zhang W, Zhu XD, Zhuang PY, Zhang JB, Wang L, Wu WZ, Qin LX, Tang ZY: Human hepatocellular carcinoma tumor-derived endothelial cells manifest increased angiogenesis capability and drug resistance compared with normal endothelial cells. Clin Cancer Res 2009, 15:4838-4846

16. Hida K, Hida Y, Amin DN, Flint AF, Panigrahy D, Morton CC, Klagsbrun M: Tumor-associated endothelial cells with cytogenetic abnormalities. Cancer Res 2004, 64:8249-8255

17. Akino T, Hida K, Hida Y, Tsuchiya K, Freedman D, Muraki C, Ohga N, Matsuda K, Akiyama K, Harabayashi T, Shinohara N, Nonomura K, Klagsbrun M, Shindoh M: Cytogenetic abnormalities of tumor-associated endothelial cells in human malignant tumors. Am J Pathol 2009, 175:2657-2667

18. Akiyama K, Ohga N, Hida Y, Kawamoto T, Sadamoto Y, Ishikawa S, Maishi N, Akino T, Kondoh M, Matsuda A, Inoue N, Shindoh M, Hida K: Tumor endothelial cells acquire drug resistance by MDR1 upregulation via VEGF signaling in tumor microenvironment. Am J Pathol 2012, 180:1283-1293

19. Ohga N, Ishikawa S, Maishi N, Akiyama K, Hida Y, Kawamoto T, Sadamoto Y, Osawa T, Yamamoto K, Kondoh M, Ohmura H, Shinohara N, Nonomura K, Shindoh M, Hida K: Heterogeneity of tumor endothelial cells: comparison between tumor endothelial cells isolated from high- and low-metastatic tumors. Am J Pathol 2012, 180: 1294-1307

20. Lasagna N, Fantappie O, Solazzo M, Morbidelli L, Marchetti S, Cipriani G, Ziche M, Mazzanti R: Hepatocyte growth factor and inducible nitric oxide synthase are involved in multidrug resistanceinduced angiogenesis in hepatocellular carcinoma cell lines. Cancer Res 2006, 66:2673-2682

21. Fidler IJ, Kim SJ, Langley RR: The role of the organ microenvironment in the biology and therapy of cancer metastasis. J Cell Biochem 2007, 101:927-936

22. Perez-Sayans M, Somoza-Martin JM, Barros-Angueira F, Diz PG, Rey JM, Garcia-Garcia A: Multidrug resistance in oral squamous cell carcinoma: the role of vacuolar ATPases. Cancer Lett 2010, 295: $135-143$

23. Kozlowski JM, Hart IR, Fidler IJ, Hanna N: A human melanoma line heterogeneous with respect to metastatic capacity in athymic nude mice. J Natl Cancer Inst 1984, 72:913-917

24. Hida K, Hida Y, Shindoh M: Understanding tumor endothelial cell abnormalities to develop ideal anti-angiogenic therapies. Cancer Sci 2008, 99:459-466

25. Osawa T, Ohga N, Hida Y, Kitayama K, Akiyama K, Onodera Y, Fujie M, Shinohara N, Shindoh M, Nonomura K, Hida K: Prostacyclin receptor in tumor endothelial cells promotes angiogenesis in an autocrine manner. Cancer Sci 2012, 103:1038-1044

26. Kurosu T, Ohga N, Hida Y, Maishi N, Akiyama K, Kakuguchi W, Kuroshima T, Kondo M, Akino T, Totsuka Y, Shindoh M, Higashino F, Hida K: HuR keeps an angiogenic switch on by stabilising mRNA of VEGF and COX-2 in tumour endothelium. Br J Cancer 2011, 104:819-829 
27. Ohga N, Hida K, Hida Y, Muraki C, Tsuchiya K, Matsuda K, Ohiro Y, Totsuka Y, Shindoh M: Inhibitory effects of epigallocatechin-3 gallate, a polyphenol in green tea, on tumor-associated endothelial cells and endothelial progenitor cells. Cancer Sci 2009, 100:1963-1970

28. Inuzuka T, Tsuda M, Kawaguchi H, Ohba Y: Transcription factor 8 activates R-Ras to regulate angiogenesis. Biochem Biophys Res Commun 2009, 379:510-513

29. Miyoshi H: Gene delivery to hematopoietic stem cells using lentiviral vectors. Methods Mol Biol 2004, 246:429-438

30. Jurado JM, Sanchez A, Pajares B, Perez E, Alonso L, Alba E: Combined oral cyclophosphamide and bevacizumab in heavily pre-treated ovarian cancer. Clin Transl Oncol 2008, 10:583-586

31. Fontana A, Galli L, Fioravanti A, Orlandi P, Galli C, Landi L, Bursi S, Allegrini G, Fontana E, Di Marsico R, Antonuzzo A, D'Arcangelo M, Danesi R, Del Tacca M, Falcone A, Bocci G: Clinical and pharmacodynamic evaluation of metronomic cyclophosphamide, celecoxib, and dexamethasone in advanced hormone-refractory prostate cancer. Clin Cancer Res 2009, 15:4954-4962

32. Bhatt RS, Merchan J, Parker R, Wu HK, Zhang L, Seery V, Heymach JV, Atkins MB, McDermott D, Sukhatme VP: A phase 2 pilot trial of low-dose, continuous infusion, or "metronomic" paclitaxel and oral celecoxib in patients with metastatic melanoma. Cancer 2010, 116:1751-1756
33. Walker L, Schalch H, King DM, Dietrich L, Eastman M, Kwak M, Kim K, Albertini MR: Phase II trial of weekly paclitaxel in patients with advanced melanoma. Melanoma Res 2005, 15:453-459

34. Kesari S, Schiff D, Doherty L, Gigas DC, Batchelor TT, Muzikansky A, O'Neill A, Drappatz J, Chen-Plotkin AS, Ramakrishna N, Weiss SE, Levy B, Bradshaw J, Kracher J, Laforme A, Black PM, Folkman J, Kieran M, Wen PY: Phase II study of metronomic chemotherapy for recurrent malignant gliomas in adults. Neuro Oncol 2007, 9:354-363

35. Krzyzanowska MK, Tannock IF, Lockwood G, Knox J, Moore M, Bjarnason GA: A phase II trial of continuous low-dose oral cyclophosphamide and celecoxib in patients with renal cell carcinoma. Cancer Chemother Pharmacol 2007, 60:135-141

36. Leenders WP, Kusters B, Verrijp $\mathrm{K}$, Maass C, Wesseling $\mathrm{P}$, Heerschap A, Ruiter D, Ryan A, de Waal R: Antiangiogenic therapy of cerebral melanoma metastases results in sustained tumor progression via vessel co-option. Clin Cancer Res 2004, 10:6222-6230

37. Bussolati B, Deambrosis I, Russo S, Deregibus MC, Camussi G: Altered angiogenesis and survival in human tumor-derived endothelial cells. FASEB J 2003, 17:1159-1161

38. Grange C, Bussolati B, Bruno S, Fonsato V, Sapino A, Camussi G: Isolation and characterization of human breast tumor-derived endothelial cells. Oncol Rep 2006, 15:381-386 